\begin{tabular}{|c|l|}
\hline Title & Nickel Nanoparticles Formation from Solution Plasma U sing Edge Shiel ded Electrode \\
\hline Author(s) & Saito, G.; Hosokai, S.; Tsubota, M.; A kiyama, T. \\
\hline Citation & $\begin{array}{l}\text { Plasma Chemistry and Plasma Processing, 31(5), 719-728 } \\
\text { https://doi.org/10.1007/311090-011-9313-4 }\end{array}$ \\
\hline Issue Date & 2011-10 \\
\hline Doc URL & http://hdl.handle.net/2115/52222 \\
\hline Rights & The original publication is available at www.springerlink.com \\
\hline Type & article (author version) \\
\hline File Information & PlaPro31_719-728.pdf \\
\hline
\end{tabular}

Instructions for use 


\title{
Nickel Nanoparticles Formation from Solution Plasma using Edge-Shielded Electrode
}

\author{
G Saito, ${ }^{1}$ S Hosokai ${ }^{1}$ M Tsubota ${ }^{1}$ and T Akiyama ${ }^{1}$ \\ ${ }^{1}$ Center for Advanced Research of Energy and Materials, \\ Faculty of Engineering, Hokkaido University, Sapporo 060-8628 \\ takiyama@eng.hokudai.ac.jp
}

\begin{abstract}
Because of the easy massproduction, synthesis of metallic nanoparticles from a solution plasma is an attractive method. However, a solution plasma produces a highly inhomogeneous electric field via transition to full-plasma, and the products are partially oxidized and agglomerated, with a wide size-distribution. Here, we show a simple method of suppressing oxidation of products. An electrode tip was shield by a glass tube and a voltage of up to $180 \mathrm{~V}$ was applied with the electrolyte of $0.1 \mathrm{M} \mathrm{NaOH}$ solution. Significantly, the edge-shield was quite effective for maintaining partially glow discharge. The results were (1) surface temperature of the electrode less than $100{ }^{\circ} \mathrm{C}$, (2) main phase of metallic nickel evaluated by XRD, and (3) nanoparticles of an average size of $220 \mathrm{~nm}$. These results showed the potential for an application to the production of nanoparticles.
\end{abstract}

Keywords. Nickel, Glow discharge, Pure metallic 


\section{Introduction}

The syntheses and applications of metallic nanoparticles have been attracting widespread attention because of the unique properties of such particles; these properties are different from those of the corresponding bulk materials because of the quantum size effect[1-4]. Among various nanoparticles, nickel (Ni) nanoparticles have a wide range of applications, such as in multi-layered ceramic capacitors[5], memory devices[6, 7], various catalysts[8, 9], and conductive pastes[10].

Plasma generation in a solution, rather than in a gas, has recently been proposed for the synthesis of nanoparticles; methods include the plasma jets[11], plasma-chemical reduction[12], microplasma reduction[13], influence of glow discharge[14], arc in water[15-18], arc discharge processes[19], solution plasma processing (SPP)[20-23], cathodic discharge[24, 25], and laser-induced plasma[26]. Some of these methods successfully produced small $(<10 \mathrm{~nm})$ uniform nanoparticles. In particular, solution plasma has been applied to the synthesis of microparticles/nanoparticles in solution[27-29]. A nickel electrode, placed at the center of a beaker, acts as the cathode in this method. Under glow discharge conditions, the surface of the nickel electrode is partially melted, forming spherical nickel nanoparticles in the solution. Solution plasma offers many advantages: (1) simple equipment, (2) no need to supply gas, (3) easy mass production, (4) applicable to any metals/alloys, and (5) easy to control a size of the product[28].

However, there is still a problem to be overcome in the case of formation of nanoparticles from the solution plasma because of transition from partial-plasma region to full-plasma one with the strong emission of light. Transition is caused by a highly inhomogeneous electric field. The products are oxidized and agglomeration after transition and post-treatments consisting of deoxidization and separation, e.g., filtration, are needed. Additionally, Toriyabe et al. reported that the product size decreased with increasing voltage, but the transition easily occurred when voltage was high. Therefore, suppression method of transition to full plasma is needed for the production of nanoparticles by solution plasma, but it still remains unsolved.

Here, we show a simple method for suppressing the transition at high voltage and study the relationship between the transition and nanoparticles. According to literature, the effect of the the transition has never been well explained. The purpose of this paper is therefore to study the effects of the transition on formation of nanoparticles in the solution plasma, in which the tip of a nickel electrode was shielded to keep a partial-plasma region, and the products were characterized using field-emission electron microscopy (FE-SEM), transmission electron microscopy (TEM) and X-ray diffractometry (XRD). Transient changes in the electrode were also monitored by a high-speed camera and a radiation thermometer. A possible mechanism for the formation of nanoparticles was finally discussed on the basis of the results obtained. 


\section{Experimental Methods}

\subsection{Experimental Setup}

Figure 1 shows the experimental apparatus used for the experiments under stable power supply conditions (ZX800H, Takasago, Tokyo, Japan) with a constant voltage supply. A platinum wire of length $1000 \mathrm{~mm}$, diameter $0.5 \mathrm{~mm}$, and purity 99.98 mass\% (Nilaco, Tokyo, Japan) was used as the anode; this wire was bent into a half-round mesh and fixed in a glass frame. A nickel wire of diameter $1.0 \mathrm{~mm}$ and purity 99 mass\% (Nilaco) was used as the cathode. It was shielded by a quartz glass tube of inner diameter $1.1 \mathrm{~mm}$ in order to obtain an exposed length of $10 \mathrm{~mm}$; the exposed part functioned as the actual electrode. We used two types of cathode: (a) without an edge-shield, or (b) with an edge-shield [see Figs. 1(a) and 1(b)]. We shielded the tip of the electrode as shown in Fig. 1(a) to suppress the transition because the transition to full-plasma was induced by the glow corona[30]. The distance between the electrodes was kept at $30 \mathrm{~mm}$. The electrolytes used were $0.1 \mathrm{M} \mathrm{NaOH}$ solutions. The solution temperature at a depth of 10 $\mathrm{mm}$ was recorded every $5 \mathrm{~s}$ using a polymer-coated thermistor thermometer (Ondotori TR-71Ui, T\&D, Nagano, Japan). The photograph of the cathode was taken using a high-speed camera (FX-FH25, CASIO, Tokyo, Japan).

\subsection{Experimental Procedure}

In the experiments, anode and cathode wire were washed by deionized water and ethanol by using an ultrasonic washer. The electrolyte was prewarmed at $60 \pm 3{ }^{\circ} \mathrm{C}$. Without prewarming of the electrolyte, the glow discharge doesn't start until the solution temperature becomes high enough to form a gas layer. The voltage was applied at a rate of $0.5 \mathrm{~V} / \mathrm{s}$ up to $180 \mathrm{~V}$ and was then maintained for $3.6 \mathrm{ks}$. Figure 1(c) shows the voltage waveform in the experiment. The voltage and current were measured by the power supply. The experiments were repeated each three times at same condition and named "No Edge-Shield-1", "No Edge-Shield-2", "No Edge-Shield-3", "Edge-Shield-1", "Edge-Shield-2" and "Edge-Shield-3" to guarantee the reproducibility. After the experiments, the products were collected by the centrifugation. The collected particles and the spent wire were washed by deionized water.

\subsection{Characterizations}

The particles were characterized by XRD using a Miniflex II (Rigaku, Tokyo, Japan) diffractometer. The surface of the cathode after the experiments and the products were observed by FE-SEM using a JSM-7001F (JEOL, Tokyo, Japan) microscope. Particle size-distributions were obtained from TEM images of the products by using a H-700 (HITACHI, Tokyo, Japan) 
microscope. To measure the particle diameter, the four samples for TEM observation were prepared. The mean diameters were evaluated on the basis of volume.

\subsection{Measurement of Surface Temperature}

The surface temperatures of the cathodes were measured using a radiation thermometer (Thermo spot FTK9-R100 sensor, Japan Sensor, Tokyo, Japan) with a measurement range from $85^{\circ} \mathrm{C}$ to $1985^{\circ} \mathrm{C}$, and a spot diameter of $0.6 \mathrm{~mm}$. In order to measure the electrode temperature directly, the cathode was folded vertically and placed on the water surface. The experiments were all conducted under the same voltage conditions, but the arrangement of the electrodes were changed. Therefore, there is a possibility that the surface temperature does not match in Fig.1. 


\section{Results and discussion}

\subsection{Suppression of transition to full-plasma by Edge-Shield}

Figure 2 shows the histories of the currents for different electrodes. The conventional electrolysis of water was performed and the current was increased with increasing the voltage when the voltage was $50 \mathrm{~V}$ or less. Since the electrical resistance heating of the solution was concentrated at the cathode/solution interface, the solution near the cathode heated to the boiling point and a gas layer containing hydrogen gas and steam was generated. Once the gas layer has been generated at the cathode surface, the current cannot then increase any further, and decreases because the cathode and the solution do not touch each other as shown in the breakdown point of current in Fig.2. If the voltage was sufficiently high, the gas layer formed a glow discharge plasma. The glow discharge with the light emission occurred at about $120 \mathrm{~V}$. The intensity of the light emission increased with increasing the voltage, which means that the net area of the discharge expanded with increasing the voltage. In this time, the discharge is a partial-plasma. Without edge-shielding, transition to full-plasma occurred at $173 \pm 2 \mathrm{~V}$ which values were evaluated in three experiments, from the edge of the nickel electrode, and emitted glare. Shortly before that, the current jumped temporarily, and then maintained a steady values of $0.51 \pm 0.01 \mathrm{~A}$. In contrast, transition to full-plasma was completely suppressed by the edge-shield. The average current decreased to $0.37 \pm 0.02 \mathrm{~A}$ in spite of same voltage when edge-shield electrode was used. The average temperature decreased to $87 \pm 1{ }^{\circ} \mathrm{C}$ from $90 \pm$ $1{ }^{\circ} \mathrm{C}$. This tendency agreed with the change of current. Figure 3 shows photographs of the exposed 10-mm part of the electrode. The upper parts of both electrodes were shielded by the quartz glass tube and the tip of the electrode was capped only in the edge-shield case. Note that at $150 \mathrm{~V}$, the cathode without an edge-shield was brighter at the edge [see Fig. 3(c)], then it ignited from the edge [see Fig. 3(d)]. In contrast, the edge-shielded cathode kept the same glow discharge, without the transition to full-plasma, even at $180 \mathrm{~V}$.

\subsection{Product Phase}

Figure 4 summarizes the XRD patterns of the products. The no-edge-shielded electrode produced both metallic nickel and nickel oxide. This was exactly the same as the result obtained in the reported data[24,25]. In contrast, the products produced by using the edge-shielded electrode didn't contain the nickel oxide. Since the powder X-ray diffractometry can't characterize the surface structure with the thickness of less than 1-2 nm, there is possibility that the particle surface was oxidized. Obviously, the transition to full-plasma observed in the former case enhanced oxidation of the product and the edge-shielded electrode was effective in suppressing the transition. The measurement of the surface temperature of the electrode 
indicated that the temperature of full plasma was more than $2000^{\circ} \mathrm{C}$, in contrast, the temperature of partial plasma was less than $100{ }^{\circ} \mathrm{C}$. The transition to full-plasma increased the surface temperature, and the products were oxidized as a result of high-temperature corrosion. In partial plasma region, the net area of plasma is small. In addition, the surface of the electrode was not uniform because of the gas generation and solution convection. Thus, the surface of the electrode cooled down by the solution. This is the reason why the electrode temperature is less than $100^{\circ} \mathrm{C}$.

\subsection{Morphology and Size}

Figure 5 shows TEM images of the products (left) and the electrode nickel needles (right). All the products were spherical, and the smallest particle-size was less than $20 \mathrm{~nm}$. The results suggested that the electrode surface was locally heated and momentarily exceeded the melting point, and the molten metal yielded to a spherical shape due to surface tension. In using no edge-shielded electrode, particles with the diameter of more than $1 \mu \mathrm{m}$ were contained and the surface of the wire showed many cracks, which were probably caused by thermal expansion due to the transition to full-plasma. Coarse particles with a diameter over $1 \mu \mathrm{m}$ attached to the electrode surface. In contrast, in using edge-shielded electrode never had any cracks or coarse particles. We also observed that regular patterns appeared on the electrode surface in the edge-shielded. This implied that nanoparticles grew at projections (see white arrows in Fig.5) and then peeled from the projections to become particles. Figure 6 shows particle size-distributions of the products which were evaluated by TEM images. The use of an edge-shielded electrode decreased the volume mean diameter of the product, to $220 \mathrm{~nm}$, in contrast to the use of an electrode without an edge-shield, where the one was $890 \mathrm{~nm}$, in which mean diameters were calculated using three dates. The suppression of coarse particles had an influence on the mean diameters. The number mean diameter was $85 \mathrm{~nm}$ in using edge-shielded electrode, which diameter was small compared to the reported data by Toriyabe et al.(2007)[27]. Toriyabe et al. reported that the product size decreased with increasing the voltage, but the transition to full-plasma easily occurred when voltage was high. Thus, the edge-shield was quite effective for the decrease of product size. Transition to full-plasma influenced not only the product size, but also nanoparticles production, which was reduced in the no-edge-shield case, as shown in Fig. 7. The mass of production after 1 hour were $15 \pm 2 \mathrm{mg}$ in no-edge-shield case, and $1.7 \pm 0.5 \mathrm{mg}$ in edge-shield case. This difference can be explained by the increasing a gas volume and a high temperature. According to the report[31], the size of the product produced by wire explosion is increased with increasing the gas volume over the cathode. Therefore, the large size of particles produced in plasmas with voltage of $180 \mathrm{~V}$ without shielding might be caused by the large volume of plasma zone as shown in Fig. 3(d). 


\subsection{Effect of transition to full-plasma}

Possible effect of transition to full-plasma was discussed on the basis of the experimental data. The glow discharge was categorized as "normal glow" or "abnormal glow"; the difference between the two discharges derived from the current [32]. The net area of plasma with "normal glow" is small compared to that of "abnormal glow"; in the latter case, the electrode surface was entirely covered with a plasma layer (full-plasma). According to the experiments results, the current increased to $0.51 \pm 0.01$ A from $0.37 \pm 0.02$ A with transition to full-plasma. In addition, a video of electrode surface taken by using a high-speed camera revealed that the net area of plasma is small before transition to full-plasma. But after the transition to full-plasma, the whole area of electrode emitted light (supplementary information available). Thus, we regard "transition to full-plasma" as the transition from "normal glow" to "abnormal glow". Without edge-shield, the edge of the electrode was heated higher than the side of the electrode since the electric field concentrated to the edge. After that, the full-plasma was generated from the edge, and it expanded to the whole area of the electrode. On the other hand, the surface of the electrode with the edge-shield was uniformly heated because of avoiding the concentration of the electric field to the edge. Partially shielding of the cathode suppressed the transition to full plasma. In using edge-shielded, the net area of plasma is small and plasma area moves quickly to keep the surface temperature low. In such case, formed nanoparticles were cool-downed quickly without agglomeration. But after transition to full-plasma, the whole area of electrode surface was always heated-up and molten metals were coalesced to form coarse particles. We assumed that the oxidation suppression by the edge-shield related with the volume of plasma zone. The plasma consisted of the steam and hydrogen. The oxide formed owing to the high temperature collusion of the steam as following equation(1).

$$
\mathrm{Ni}+\mathrm{H}_{2} \mathrm{O}=\mathrm{NiO}+\mathrm{H}_{2}(\mathrm{~g})
$$

The full-plasma has the large volume of plasma zone to produce oxide particles. In the partial-plasma region, the molten metal immediately cooled down to produce metallic particles. 


\section{Conclusions}

In this paper, the relationship between the transition to full-plasma and nanoparticles formation via a solution plasma was studied experimentally, with the following conclusions:

1. The electrode edge-shield successfully prevented the transition to full-plasma in the solution plasma.

2. The method was effective in avoiding oxidation of the products. XRD analysis showed that the main phase of the products was a metallic nickel. In contrast, the products obtained with the transition to full-plasma were nickel and nickel oxide.

3. The method also decreased the size of the products. TEM showed that the mean diameter was $220 \mathrm{~nm}$ for the product in partial-plasma, in contrast to $890 \mathrm{~nm}$ for the product in full-plasma.

4. Without the edge-shield, the surface of the electrode after the experiment was rough, with many cracks; this was probably caused by the extremely high temperature, together with formation of coarse particles near the cracks. In contrast, the electrode with the edge-shield had a surface temperature less than $100{ }^{\circ} \mathrm{C}$, and the surface of the electrode was quite smooth. This was why the edge-shield produced smaller products.

We hope that these findings will lead to further developments in the use of solution plasma for nanoparticles synthesis. Such methods have the benefits of easy mass production and wide application to conductive materials such as stainless steel. 


\section{References}

[1] Zheng X, Kubozono H, Yamada H, Kato K, Ishiwata Y and Xu C 2008 Giant negative thermal expansion in magnetic nanocrystals Nature Nanotechnology $\mathbf{3}$ 724-6

[2] Satoh N, Nakashima T, Kamikura K and Yamamoto K 2008 Quantum size effect in $\mathrm{TiO} 2$ nanoparticles prepared by finely controlled metal assembly on dendrimer templates Nat Nano 3 106-11

[3] Kubo R 1962 Electronic Properties of Metallic Fine Particles. I J. Phys. Soc. Jpn. 17975

[4] Kawabata A and Kubo R 1966 Electronic properties of fine metallic particles. II. Plasma resonance absorption J. Phys. Soc. Jpn. 211765

[5] Chen W, Li L, Qi J, Wang Y and Gui Z 1998 Influence of Electroless Nickel Plating on Multilayer Ceramic Capacitors and the Implications for Reliability in Multilayer Ceramic Capacitors J. Am. Ceram. Soc. 81 2751-2

[6] Gong J, Wang L L, Liu Y, Yang J H and Zong Z G 2008 Structural and magnetic properties of hcp and fcc Ni nanoparticles J. Alloys Compd. 457 6-9

[7] Mourdikoudis S, Simeonidis K, Vilalta-Clemente A, Tuna F, Tsiaoussis I, Angelakeris M, Dendrinou-Samara C and Kalogirou O 2009 Controlling the crystal structure of Ni nanoparticles by the use of alkylamines J. Magn. Magn. Mater. 321 2723-8

[8] Jeng Y-R, Wen H-C and Tsai P-C The effect of Ni catalytic nanoparticle on the growth of carbon nanotubes: A perspective from nanotribological characterization Diamond Relat. Mater. 18 528-32

[9] Chen C-Y, Lin K-Y, Tsai W-T, Chang J-K and Tseng C-M 2010 Electroless deposition of $\mathrm{Ni}$ nanoparticles on carbon nanotubes with the aid of supercritical $\mathrm{CO} 2$ fluid and a synergistic hydrogen storage property of the composite Int. J. Hydrogen Energy 35 5490-7

[10] Kim S-G, Terashi Y, Purwanto A and Okuyama K 2009 Synthesis and film deposition of $\mathrm{Ni}$ nanoparticles for base metal electrode applications Colloids Surf., A 337 96-101

[11] Furusho H, Kitano K, Hamaguchi S and Nagasaki Y 2009 Preparation of Stable Water-Dispersible PEGylated Gold Nanoparticles Assisted by Nonequilibrium Atmospheric-Pressure Plasma Jets Chem. Mater. 21 3526-35 
[12] Koo I G, Lee M S, Shim J H, Ahn J H and Lee W M 2005 Platinum nanoparticles prepared by a plasma-chemical reduction method J. Mater. Chem. 15 4125-8

[13] Richmonds C and Sankaran R M 2008 Plasma-liquid electrochemistry: Rapid synthesis of colloidal metal nanoparticles by microplasma reduction of aqueous cations Applied Physics Letters 93131501

[14] Liang X, Wang Z-j and Liu C-j 2010 Size-Controlled Synthesis of Colloidal Gold Nanoparticles at Room Temperature Under the Influence of Glow Discharge Nanoscale Res. Lett. 5 124-9

[15] Sano N, Charinpanitkul T, Kanki T and Tanthapanichakoon W 2004 Controlled synthesis of carbon nanoparticles by arc in water method with forced convective jet J. Appl. Phys. 96 645-9

[16] Muthakarn P, Sano N, Charinpanitkul T, Tanthapanichakoon W and Kanki T 2006 Characteristics of Carbon Nanoparticles Synthesized by a Submerged Arc in Alcohols, Alkanes, and Aromatics J. Phys. Chem. B 110 18299-306

[17] Kawanami O and Sano N 2009 Gravitational Effects on Carbon Nano-Materials Synthesized by Arc in Water Ann. N.Y. Acad. Sci. 1161 494-9

[18] Li H, Guan L, Shi Z and Gu Z 2004 Direct Synthesis of High Purity Single-Walled Carbon Nanotube Fibers by Arc Discharge J. Phys. Chem. B 108 4573-5

[19] Yao W-T, Yu S-H, Zhou Y, Jiang J, Wu Q-S, Zhang L and Jiang J 2005 Formation of Uniform $\mathrm{CuO}$ Nanorods by Spontaneous Aggregation: Selective Synthesis of $\mathrm{CuO}, \mathrm{Cu} 2 \mathrm{O}$, and $\mathrm{Cu}$ Nanoparticles by a Solid-Liquid Phase Arc Discharge Process J. Phys. Chem. B 109 14011-6

[20] Takai O 2008 Solution plasma processing (SPP) Pure Appl. Chem. 80 2003-11

[21] Ichin Y, Mitamura K, Saito N and Takai O 2009 Characterization of platinum catalyst supported on carbon nanoballs prepared by solution plasma processing $J$. Vac. Sci. Technol., A 27826

[22] Saito N, Hieda J and Takai O 2009 Synthesis process of gold nanoparticles in solution plasma Thin Solid Films 518 912-7

[23] Kim S M, Kim G S and Lee S Y 2008 Effects of PVP and KCl concentrations on the synthesis of gold nanoparticles using a solution plasma processing Mater. Lett. 62 4354-6

[24] Tokushige M, Nishikiori T and Ito Y 2010 Formation of Fine Ni Nanoparticle by Plasma-Induced Cathodic Discharge Electrolysis Using Rotating Disk Anode J. Electrochem. Soc. 157 E162-E6 
[25] Tokushige M, Nishikiori T and Ito Y 2010 Plasma-induced cathodic discharge electrolysis to form various metal/alloy nanoparticles Russ. J. Electrochem. 46 619-26

[26] Yonezawa T, Hyono A, Sato S and Ariyada O 2010 Preparation of Zinc Oxide Nanoparticles by Using Microwave-induced Plasma in Liquid Chem. Lett. 39 783-5

[27] Toriyabe Y, Watanabe S, Yatsu S, Shibayama T and Mizuno T 2007 Controlled formation of metallic nanoballs during plasma electrolysis Appl. Phys. Lett. 91 041501-3

[28] Saito G, Hosokai S, Akiyama T, Yoshida S, Yatsu S and Watanabe S 2010 Size-Controlled Ni Nanoparticles Formation by Solution Glow Discharge $J$. Phys. Soc. Jpn. 79083501

[29] Saito G, Hosokai S, Tsubota M and Akiyama T 2011 Synthesis of copper/copper oxide nanoparticles by solution plasma J. Appl. Phys. 110023302

[30] Chang J S, Lawless P A and Yamamoto T 1991 Corona discharge processes IEEE Trans. Plasma Sci. 19 1152-66

[31] Cho C, Choi Y, Kang C and Lee G 2007 Effects of the medium on synthesis of nanopowders by wire explosion process Applied Physics Letters 91141501

[32] Roth J, Rahel J, Dai X and Sherman D 2005 The physics and phenomenology of One Atmosphere Uniform Glow Discharge Plasma (OAUGDP(tm)) reactors for surface treatment applications J. Phys. D: Appl. Phys. 38555 
Figures
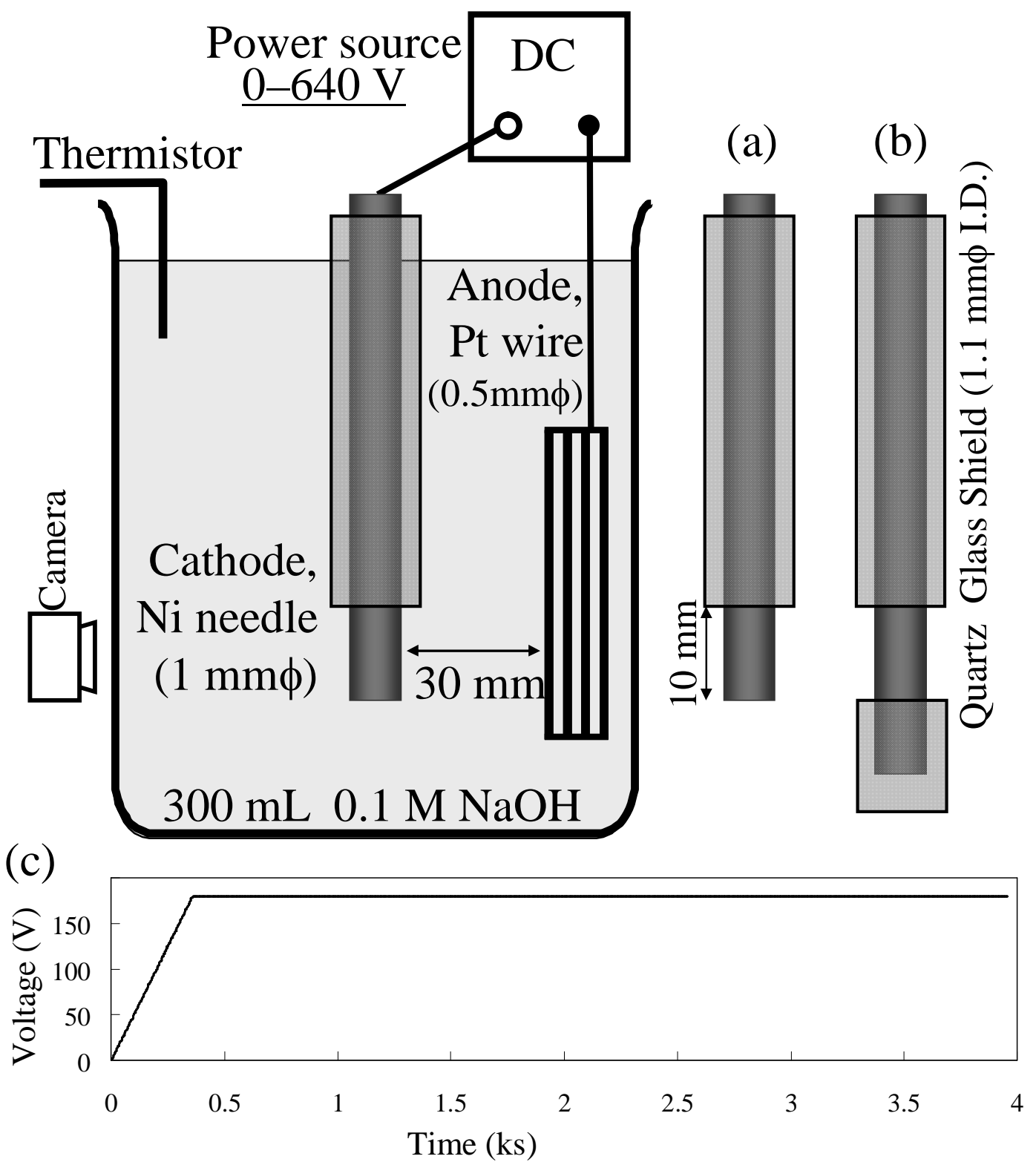

Figure 1. Schematic diagram of the experimental apparatus. As the cathode, one of two nickel needles, (a) no edge-shield, or (b) with an edge-shield, was immersed and a voltage was applied up to $180 \mathrm{~V}$. In case (b), the shield resulted in only an exposed part of length $10 \mathrm{~mm}$ acting as an electrode. The anode was a platinum wire of length $1000 \mathrm{~mm}$ and diameter $0.5 \mathrm{~mm}$; the distance between the electrodes was kept at $30 \mathrm{~mm}$. (c) voltage waveform of the experiment. 


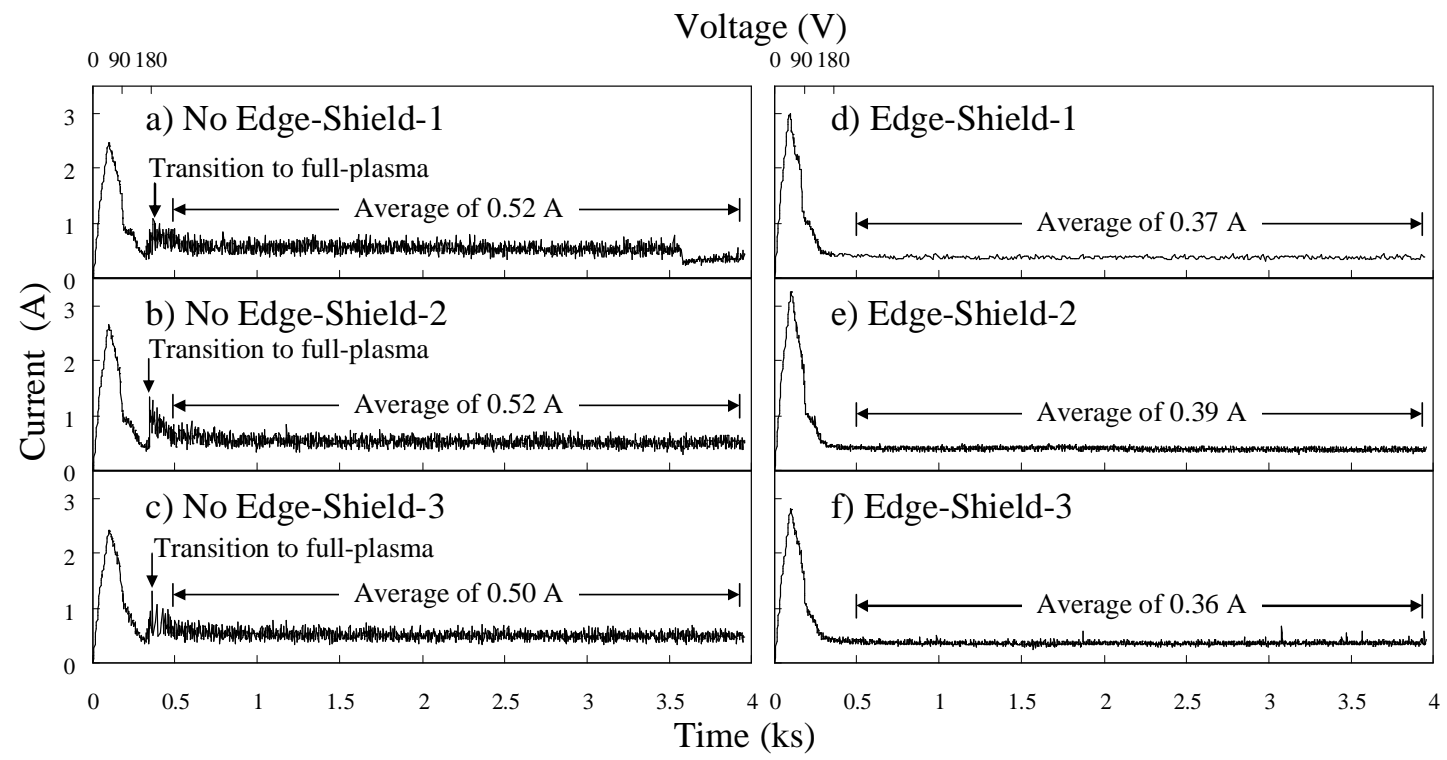

Figure 2 Histories of current. The voltage was applied at a rate of $0.5 \mathrm{~V} / \mathrm{s}$ up to $180 \mathrm{~V}$ and was then was maintained for $3.6 \mathrm{ks}$. [(a)-(c)] The transition to full-plasma from the electrode tip occurred at $173 \pm 2 \mathrm{~V}$. The average current was $0.51 \pm 0.01 \mathrm{~A}$. [(d)-(f)] The transition to full-plasma was suppressed by the edge-shield. The average current was $0.37 \pm 0.02 \mathrm{~A}$. 


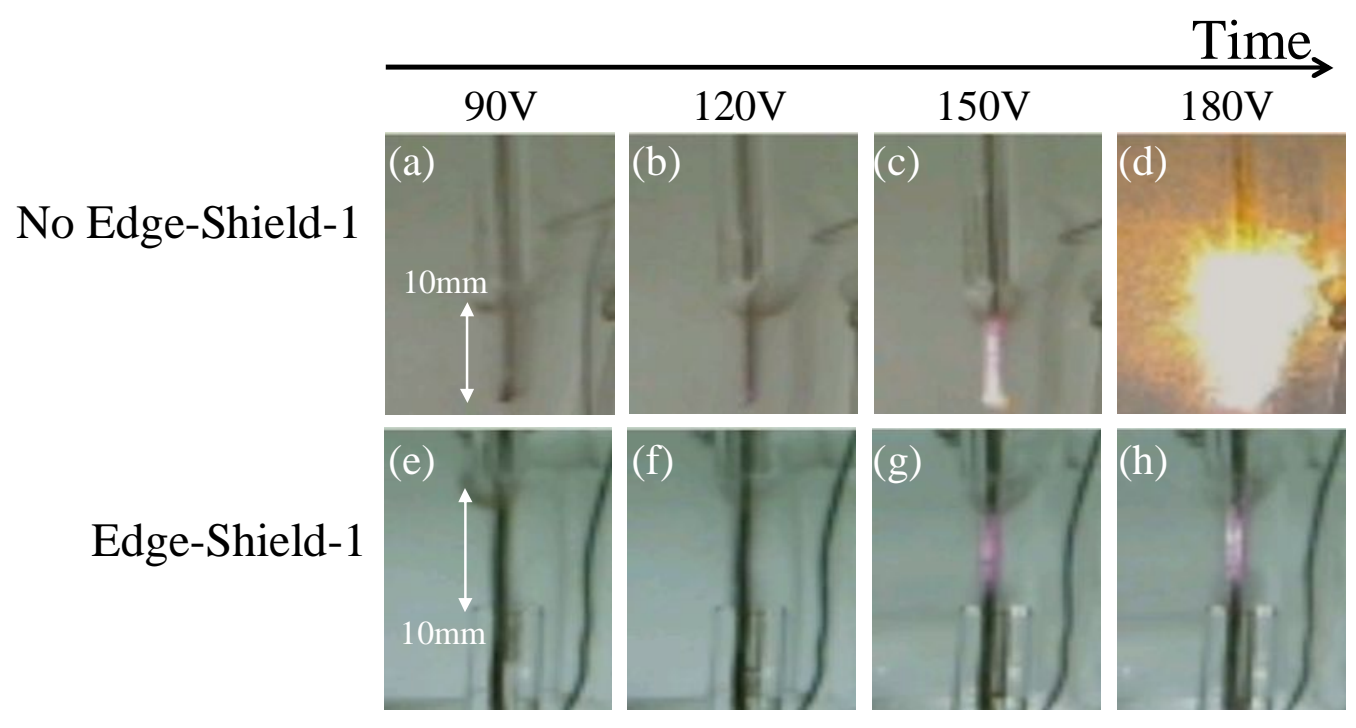

Figure 3 shows photographs of the exposed 10-mm part of the electrode. The upper parts of both electrodes were shielded by the quartz glass tube and the tip of the electrode was capped only in the edge-shield case. Note that at $150 \mathrm{~V}$, the cathode without an edge-shield was brighter at the edge [see Fig. 3(c)], then it ignited from the edge [see Fig. 3(d)]. In contrast, interestingly, the edge-shielded cathode kept the partial glow discharge, without transition to full-plasma, even at $180 \mathrm{~V}$. 


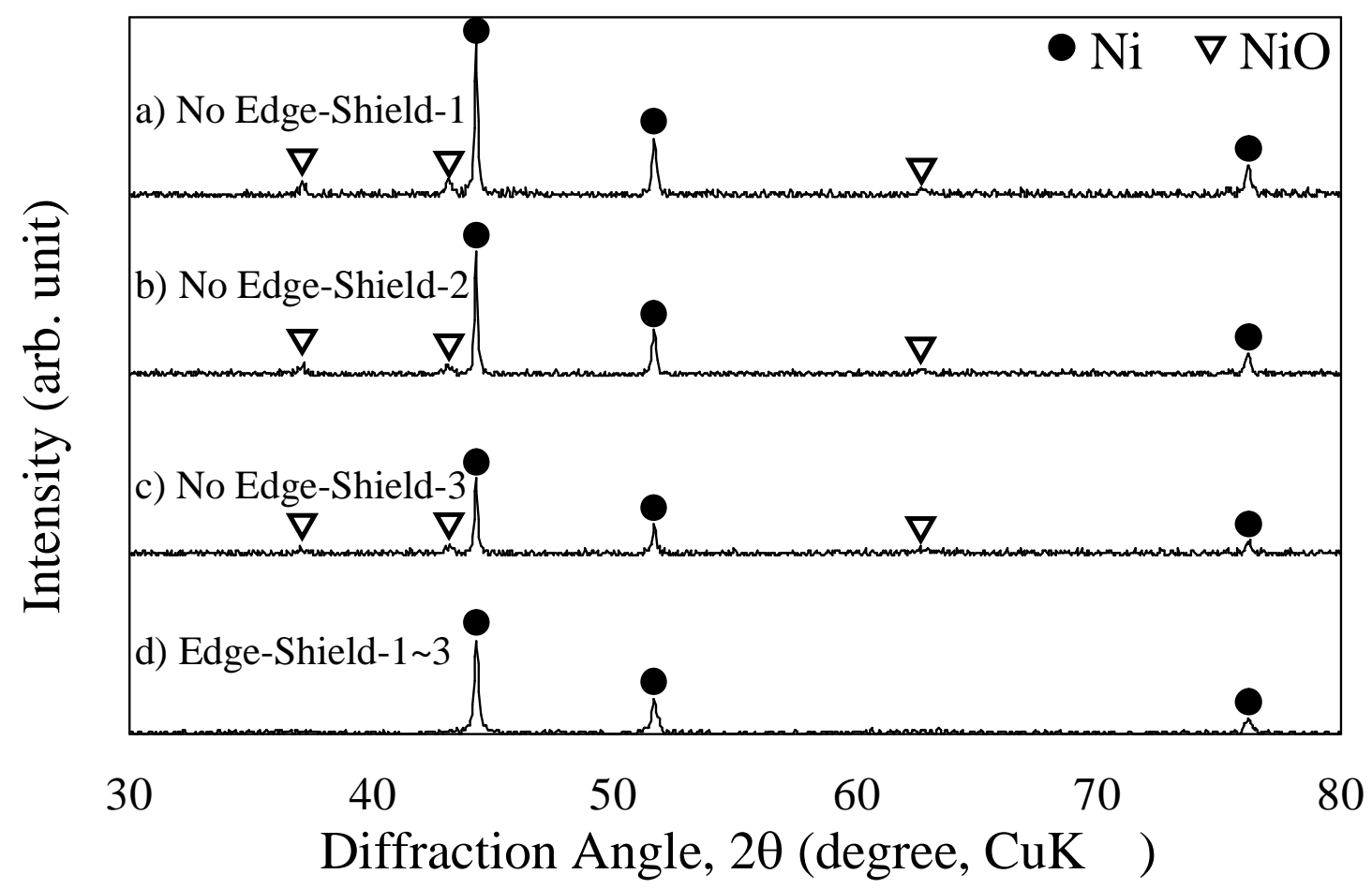

Figure 4 XRD patterns of the products from the two cathodes without the edge shielded (a)-(c), and with the edge shielded (d). The no-edge-shielded electrode produced both metallic nickel and nickel oxide. In contrast, the products produced by using the edge-shielded electrode didn't contain the nickel oxide. 


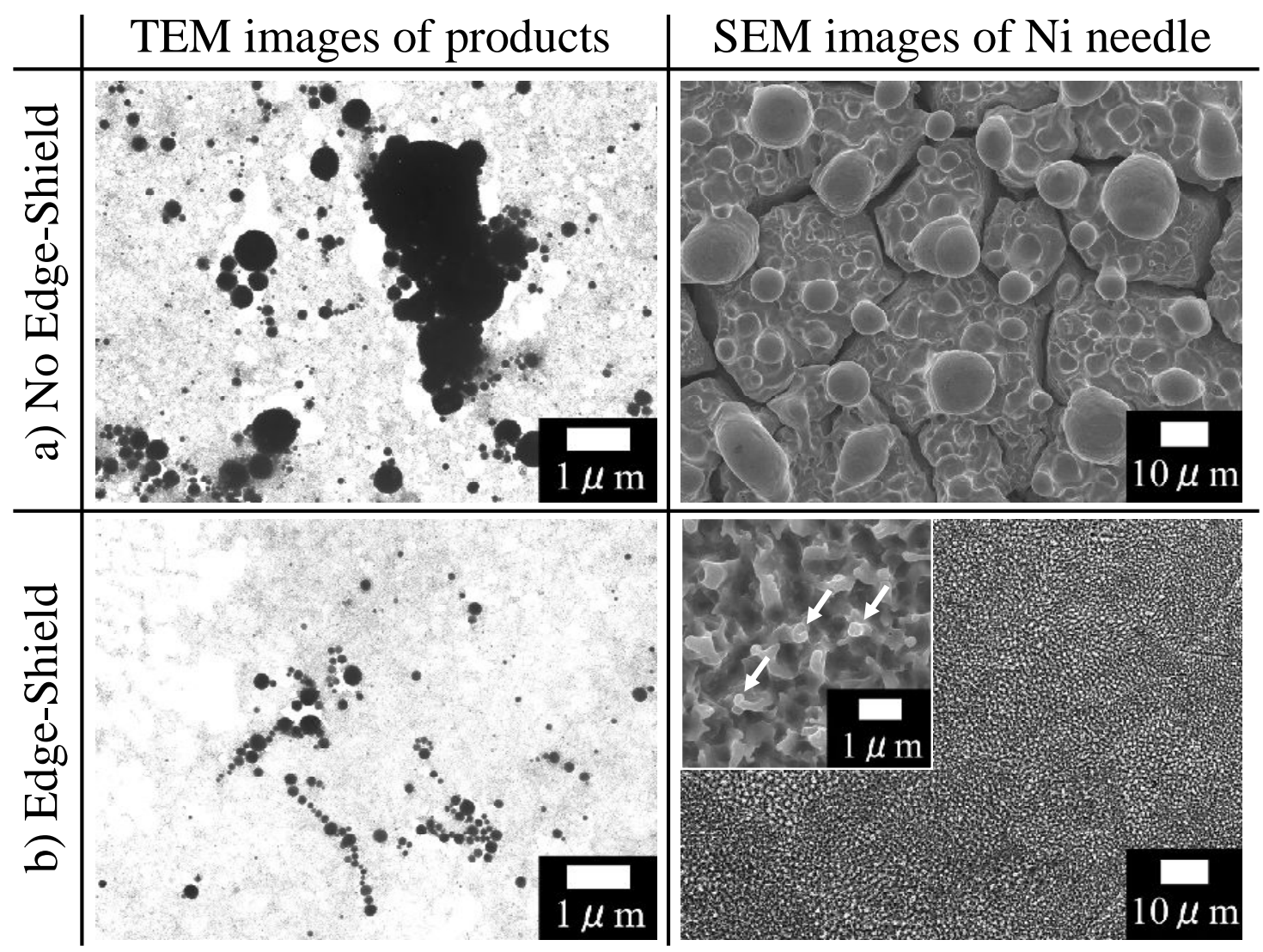

Figure 5 TEM images of the product (left) and SEM images of the surface of the nickel needle (right) when the edge-shield was used/not used. All the products were spherical, and the smallest particle-size was less than $20 \mathrm{~nm}$. 


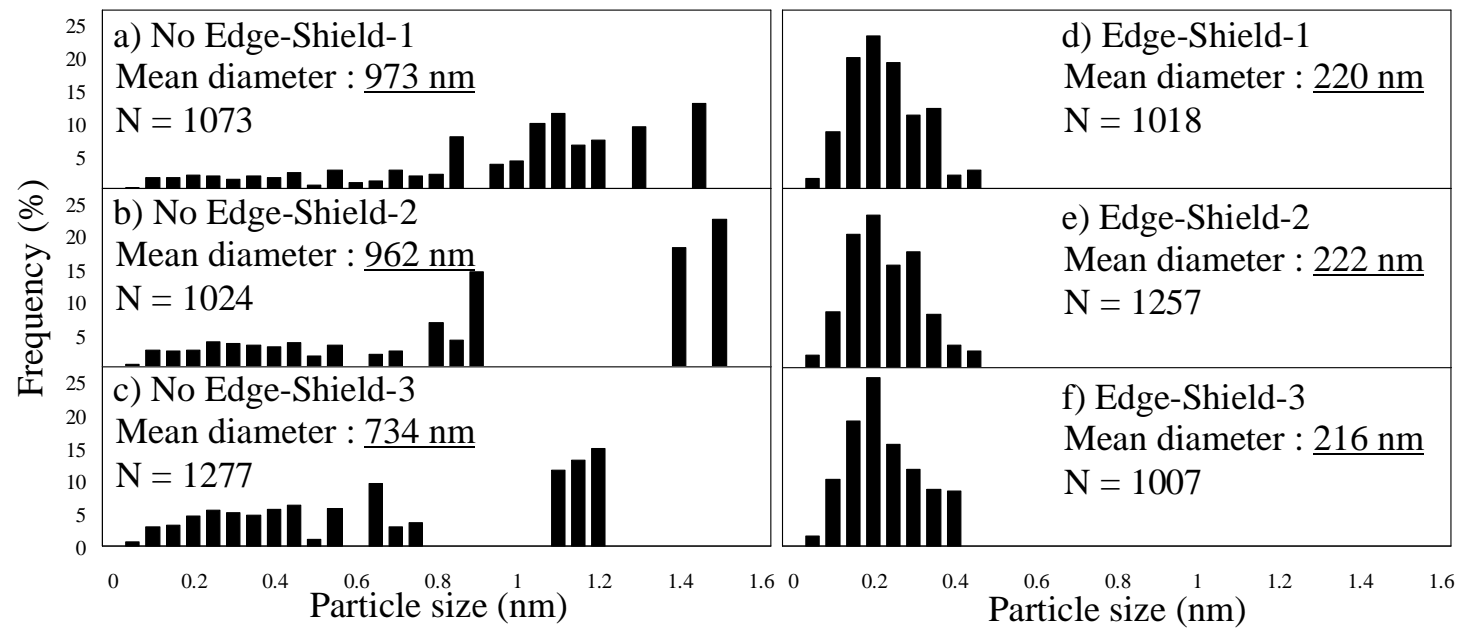

Figure 6 Particle size-distributions of the products, measured from TEM images of the products, together with the mean diameter based on volume and the sample number $\mathrm{N}$. The use of an edge-shielded electrode decreased the volume mean diameter of the product, to $220 \mathrm{~nm}$, in contrast to the use of an electrode without an edge-shield, where the one was $890 \mathrm{~nm}$, in which mean diameters were calculated using three dates.

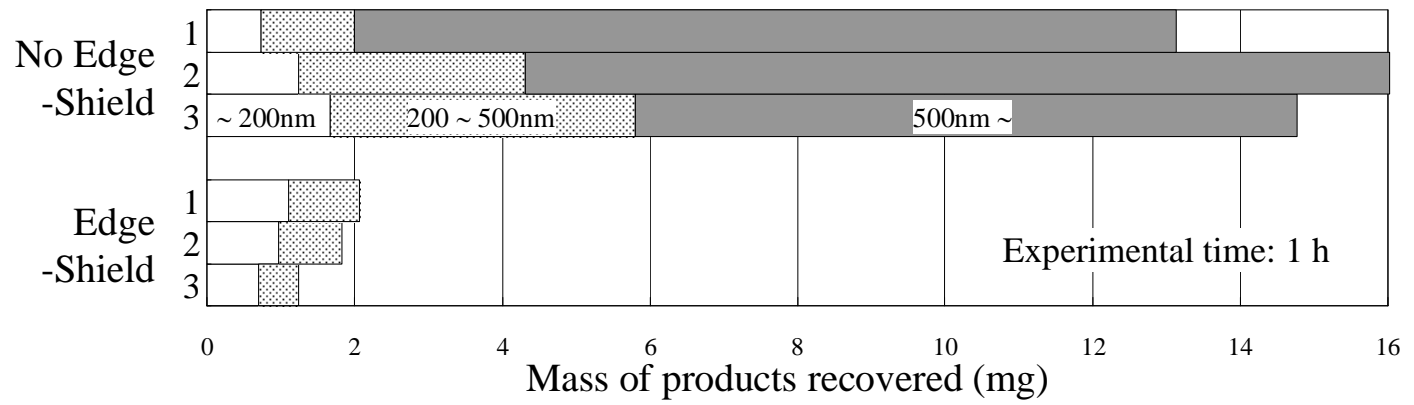

Figure 7 mass of products recovered after 1 hour were $15 \pm 2 \mathrm{mg}$ in no-edge-shield case, and 1.7 $\pm 0.5 \mathrm{mg}$ in edge-shield case. Large particles of diameter over $500 \mathrm{~nm}$ reached as much as $73 \pm$ 12 mass \% without the edge-shield. 